\title{
La formación docente desde la percepción de los egresados de una universidad pedagógica
}

\section{Teacher training from the graduated perception of a pedagogical university}

\section{Sara Lara}

saralaradegonzalez@hotmail.com

Universidad Experimental Pedagógica Libertador. Instituto Pedagógico de Caracas. Venezuela

\section{RESUMEN}

El objetivo de la investigación fue Interpretar a través de la reflexión de los egresados, sus experiencias en torno a la formación docente recibida y la transferencia realizada en los entornos educativos donde se desempeñan. Como investigación Cualitativa, se aplicó el Método Comparativo Continuo de la Teoría Fundamentada a las entrevistas en profundidad realizadas a docentes egresados de una Universidad pedagógica. Se concluyó que el estudio de las reflexiones de los egresados en torno a su formación docente, se constituye en fuente de información válida que puede servir en insumo para las instituciones formadoras de formadores a fin de mejorar la formación impartida. Se requiere Formar para a) Orientar los problemas del contexto educativo b) Utilizar adecuadamente las herramientas tecnológicas c) Enfrentar la obsolescencia del conocimiento y d) Formar desde la civilidad apegados a los valores de democracia y libertad en la búsqueda de la dignificación de la profesión docente.

Palabras clave: educación; formación docente; reflexión docente

\begin{abstract}
The objective of the research was to interpret, through the reflection of the graduates, their experiences regarding the teacher training received and the transfer made in the educational environments where they work. As Qualitative research, the Continuous Comparative Method of Grounded Theory was applied to in-depth interviews with teachers graduated from a Pedagogical University. It was concluded that the study of the reflections of graduates regarding their teacher training, is a source of valid information that can serve as an input for the training institutions of trainers in order to improve the training provided. Formation is required to a) Orient the problems of the educational context b) Use the technological tools properly c) Confront the obsolescence of knowledge and d) Form from civility attached to the values of democracy and freedom in the search for the dignity of the teaching profession.
\end{abstract}

Key words: training teacher; qualitative research; comparative continuous method 


\section{INTRODUCCIÓN}

Entre algunas de las certezas compartidas en el mundo educativo, podemos mencionar, entre otras, que la calidad de la educación se sostiene en la formación de sus docentes, la cual a menudo está relacionada con una buena inversión por parte de los organismos públicos o privados. Adicionalmente, existe la necesidad de mancomunar esfuerzos de transformación por parte de las administraciones para adecuarla y reformularla ante los constantes cambios experimentados por la sociedad actual. Por ello, se realizan esfuerzos en las diferentes universidades, dirigidos a la búsqueda de alternativas para lograr una mayor eficiencia en la formación de los docentes y estar a la altura de las nuevas exigencias que depara la multidimensionalidad del mundo complejo en el cual vivimos.

En este sentido la UNESCO, (2009) señala: "La Educación Superior amplía la formación de los profesores con planes y programas de estudios que den a los docentes la capacidad de dotar a sus alumnos de los conocimientos $\mathrm{y}$ las competencias que necesitan en el siglo XXI". No obstante, en la revisión de la literatura reciente nos encontramos con críticas diversas acerca de la formación recibida por los docentes, pues esta no siempre llega con efectividad a los espacios naturales del proceso educativo, (Gómez, Ortiz y González (2017).

Esto implica una necesidad de prever tiempos y espacios a ser utilizados por el profesorado a fin de conocer los avances en el conocimiento, las nuevas tendencias pedagógicas centradas en el estudiante, las estrategias relacionadas con las inteligencias múltiples, el aprendizaje para la autonomía y ser ciudadano del mundo. Así como también, ampliar su rol profesional en respuesta a la utilización de las tecnologías de la información y en el abordaje de las necesidades sociales del entorno educativo. En suma, tiempo y espacio destinado a resignificar formas abiertas de ver el mundo y ampliar los límites alcanzados durante los estudios del pregrado. La formación docente en estos términos argumentan Padilla, López y Rodríguez (2015), implica asumirla como un proceso continuo, sistémico, flexible, orientado a preparar al docente para la intervención en los procesos educativos a los cuales se vincula, de manera que puedan satisfacer las exigencias del cumplimento de los objetivos de formación del profesional, según la especificidad formativa.

En este sentido, Bozu e Imbernón (2016), señalan que el concepto de formación a lo largo de toda la vida (lifelong learning) implica una redefinición de la universidad como formación básica que sienta los cimientos para seguir aprendiendo y no como etapa final de formación

No obstante, en el discurso protocolar de los actos de grado universitario es común escuchar: estamos orgullosos de haber contribuido a la formación profesional de este grupo de graduando. Pareciera que el compromiso de la Universidad termina en ese importante momento, pero... ¿Qué tan preparado estará ese nuevo profesional, más allá de lo académico, para afrontar el diario trajinar del complejo mundo de la educación, solapado tras las fachadas de las instituciones educativas? ¿Qué piensan los egresados de la formación recibida? ¿De qué depende una buena formación docente?

En función de estas preguntas nos trazamos el objetivo del trabajo: Interpretar a través de la reflexión de los egresados, sus experiencias en torno a la formación docente recibida y la transferencia realizada en los entornos educativos donde se desempeñan.

La presentación está organizada en cinco secciones: 
Introducción, donde se indica el problema y las preguntas de investigación, los objetivos a lograr, la vía metodológica a seguir.

- El contexto o marco referencial, donde se revisan los postulados teóricos de los diferentes autores que se han pronunciado en torno a la formación docente $\mathrm{y}$ al seguimiento del egresado

- Método o camino a transitar, en el mismo se establecen los argumentos por los cuales la investigación es de carácter cualitativo y, la escogencia de la reflexión como vía para la construcción de conocimiento.

- Los hallazgos desde las reflexiones contenidas en las bitácoras, cuya interpretación se hace en función de la aplicación del Método Comparativo Continuo de la Teoría Fundamentada según Strauss y Corbin (2002).

- Conclusiones, reflexiones finales. El desarrollo conduce al aparte subtitulado: Nuevo mapa pedagógico, donde incluimos las conclusiones desde la provisionalidad como característica de la producción intelectual cualitativa.

\section{El contexto o marco referencial}

Lo asumiremos en tres planos: uno de ellos para referenciar a) cómo se entiende el término formación en general y luego de manera más específica, la formación docente, b) algunas investigaciones realizadas en torno a la relación entre formación docente seguimiento al egresado y c) la presentación de los postulados que permiten acudir a la reflexión como vía para la interpretación de las experiencias de los docentes entrevistados.

Ya hemos indicado en la introducción que entenderemos por formación docente tanto la formación inicial como la formación en servicio, esta última también conocida como perfeccionamiento, formación permanente, continua, actualización, acompaña-miento, desarrollo profesional y capacitación. Con respecto a este último, Rodríguez (2009) argumenta que en la práctica el vocablo capacitación a menudo sustituye a cualquiera de los mencionados pero su connotación es restringida pues implica una información lineal, seguimiento de instrucciones cuyo espíritu dista de un verdadero desarrollo profesional.

Por su parte, Díaz (2006) expresa que la formación docente incluye a la formación académica recibida en las instituciones de educación superior, la formación adquirida en el ejercicio de la profesión docente y en las múltiples relaciones contextuales que se dan en la sociedad.

Veamos ahora cómo es entendido este término en otras latitudes; en alemán, el vocablo formación, bildung, está estrechamente vinculado a las ideas de enseñanza, aprendizaje y competencia personal, significa también la cultura de una persona como resultado de su apropiación de los contenidos ofrecidos por su entorno, de algo más elevado e interior, indica Gadamer (1999), que se trata de una actitud espiritual que procede del conocimiento, del sentimiento de la vida espiritual y ética la cual fluye armónicamente en la sensibilidad y el carácter (p.40). En función de lo anterior, la formación, sólo puede ser buscada en el acto reflexivo del educador, pues en la formación, la persona se apropia por entero de aquello en lo cual y a través de lo cual se forma. En realidad, sostiene el filósofo, al dialogar nos formamos en la comprensión (p.45), ya que comprender es un aspecto propio de la formación pues, sólo la persona formada es capaz de comprender al otro, de lograr un entendí-miento. 
Estos postulados gadamerianos son pertinentes para la formación integral de los formadores de formadores en una sociedad tan convulsionada como la actual. En múltiples ocasiones, estas convulsiones sociales son parte de distorsiones que alcanzan al sector educativo. En estas condiciones es importante recordar a Yegres (2017) quien apunta que la educación universitaria debe tener un propósito democrático que prevalezca por sobre otro cualquiera, a fin de reforzar la dimensión humana en cada individuo y nos conmina a preguntarnos: ¿Se educa para la sumisión y la opresión o se educa para la libertad y la democracia? Para abordar el aprendizaje en tales circunstancias, los investigadores indican la necesidad de promover capacidades y competencias en la formación de los docentes, formar para la autonomía, la responsabilidad social y ética del ciudadano.

En este sentido, como lo establece Jiménez (2014), participamos de una sociedad caracterizada por el avance arrollador de las TIC, abierta y compleja, de nuevas demandas educativas y sociales de la sociedad cognitiva, sociedad de la información, del conocimiento múltiple, de la conciencia de la caducidad del mismo, complejidad, conflicto, riesgos e incertidumbres, todo lo cual impone la formación continua lo largo de la vida.

Visto de esta manera, la formación es permanente e implica cambios en el comportamiento del individuo a través de la mediación en un contexto determinado. Es un proceso continuo de adquisición de aprendizajes, de preparación para ejercer de una manera integral las potencialidades cognitivas lo cual propicia la creación intelectual, la vinculación social que permite desarrollar habilidades, capacidades, estrategias para constituirse en herramientas de mejora-miento de sí mismo y de transformación social de su entorno. De este modo, los docentes son piezas fundamentales de cambio en el transitar hacia la calidad, porque de su desempeño depende el éxito de las políticas educativas lo cual implica la necesidad de reformular y adecuar la educación a los tiempos que estamos viviendo.

Cuando revisamos estudios relacionados con el seguimiento al egresado, en torno a la formación docente inicial, encontramos que las universidades le han asignado una importancia investigativa menor de la esperada. En relación con este último aspecto Gómez, Ortiz y González (2017) precisan que no se trata solamente de conocer la ubicación de los egresados en el mundo laboral sino de constatar si la Universidad está cumpliendo con las demandas de la sociedad y a partir de ello retroalimentarse y mejorar.

Ruiz y Sequeira (2010) ponderan las investigaciones acerca del seguimiento al egresado, por las posibilidades de cambio que se podrían generar a partir de sus análisis en reuniones técnicas, congresos educativos entre otros, a fin de elevarlas a las instancias pertinentes con facultades de decisión.

Manteniendo como telón de fondo los argumentos de los investigadores referenciados en el aparte anterior, procederemos a revisar los postulados teóricos que sustentan la reflexión como fuente de conocimiento; ya que es el camino a transitar para interpretar los datos empíricos proporcionados por los protagonistas de este trabajo.

\section{La reflexión como fuente de conocimiento.}

La actitud reflexiva, como proceso para generar conocimiento, ha sido admitido desde 1933 por Dewey (1989), quien sostenía que reflexionar implica mirar hacia atrás y contemplar lo que se ha hecho, con objeto de extraer los significados netos a fin de utilizarlos en experiencias futuras; la función del pensamiento reflexivo (...) es la de 
transformar una situación en la que se experimenta oscuridad, duda, conflicto, o algún tipo de perturbación, en una situación clara, estable y armoniosa.

La reflexión la asume Van Manen (1997), como una toma de conciencia y para diferenciarla, propuso tres niveles: técnica, práctica y crítica. El primer nivel, se refiere a la valoración de la eficacia de los medios para lograr fines educativos, los cuales no están sujetos a crítica. El segundo, permite el examen no sólo de los medios sino también de los objetivos y de los presupuestos en los que se basan unos y otros. El tercer nivel, además de incorporar preocupaciones de los dos niveles anteriores, incluye también consideraciones de tipo moral o ético y de valoración en el sentido de si la actividad profesional es equitativa, justa y respetuosa con las personas. La reflexión crítica, además, sitúa el análisis de la acción personal en un contexto socio-histórico y político-cultural.

De este modo, la reflexión se constituye en dimensión para interpretar las situaciones cotidianas verbalizadas, por los informantes clave.

\section{El camino a transitar}

\section{MÉTODO}

Esta investigación se fundamenta en el paradigma Interpretativo donde la realidad social se constituye en el objeto central de los procesos de investigación. Azpúrua (2004) expone: el mundo social puede ser descrito tal y como es encontrado por los que en este viven.

La escogencia de los informantes estuvo, conformada por 10 (diez) personas. Los criterios de selección fueron: a) ser profesores egresados de la UPEL de diferentes especialidades, b) tener entre 1- 5 años de egreso. Por otra parte, la investigadora se incluye, como observadora participante, tal y como lo propicia la investigación cualitativa.
Se utilizó la técnica de la entrevista en profundidad, siguiendo los criterios establecidos por Taylor y Bogdan (1987).

Para analizar dichas entrevistas, se aplicó el Método Comparativo Continuo de la Teoría Fundamentada de acuerdo con Strauss y Corbin (2002): a) transcripción detallada de los contenidos de las bitácoras, b) segmentación de los contenidos en unidades de información significativa, c) clasificación o codificación de cada unidad de información d) categorización abierta, axial y selectiva e) contrastación de fuentes y f) redacción de la síntesis conceptual.

En la codificación, los sucesos, incidentes, expresados en los testimonios son agrupados en categorías, conceptos o constructos; así los datos se analizan, comparan e integran de manera inductiva y deductiva en un juego dialéctico para configurar una comprensión del hecho estudiado, a través de operaciones analíticas de codificación y así obtener teorizaciones sustantivas.

\section{Los hallazgos contenidos en las bitácoras}

A continuación se incluyen las diferentes unidades de información que la autora ha considerado pertinente compartir para los propósitos del estudio.

...la formación docente la entiendo como un proceso continuo e integral con el fin de desarrollar las capacidades, conocimientos, estrategias, herramientas necesarias para ejercer de forma idónea la labor educativa. Tal labor resulta sistémica, por cuanto se requiere de habilidades de distintos ámbitos, que incluye el área del conocimiento abordado, los aspectos pedagógicos, didácticos y el conocimiento biopsicosocial del estudiante atendido. 
El docente reflexiona con claridad conceptual acerca de su rol. De su verbalización emerge la subcategoría multiplicidad de funciones la cual define la formación docente de modo que este se constituye en un profesional polifacético para abordar la multidimensionalidad de los aspectos que el contexto le impone en su diario accionar. Díaz (2006), la define como un proceso complejo vista su naturaleza humana, de orientación pedagógica donde se evidencia la orientación formal e informal dominios pedagógicos, didácticos, disciplinares, éticos y estéticos que interactúan en un contexto multireferencial.

...en los liceos públicos encuentras consumo de drogas, alcohol, maltrato familiar, alumnos, embarazo de adolescentes, etc. Situaciones adversas y no tienes herramientas para lidiar con eso; uno se siente muy inseguro en el ejercicio de la profesión. Dejé de trabajar en un público porque hay mucha violencia, sentí que no estaba preparada para manejar esos problemas, prefiero el privado

De la verbalización del informante surge la subcategoría formación alejada de la realidad social del país vemos como la carencia de formación para manejar los problemas generados por la cruda realidad del contexto socioeconómico y cultural, impacta los contextos críticos de escasas oportunidades de crecimiento. Esto conduce a una situación deficitaria en cuanto a la presencia de la institución educativa, así como también, de la calidad de los esfuerzos de los docentes como de los aprendizajes de los estudiantes. Lamentablemente, cuando los docentes evaden laborar en estos sectores educativos, se erosiona el crecimiento y la sostenibilidad del desarrollo socio económico de un país. La violencia estudiantil trasciende nuestras fronteras. Sin embargo, en
Venezuela se ha intensificado peligrosamente en las últimas décadas y muchas veces estimulado por aquellos llamados a servir de modelos ciudadanos

...en cuanto a contenidos disciplinares
nuestra formación docente univer-
sitaria es mejor si la comparamos con
otro profesional graduado de otra
universidad, como las creadas
recientemente con sesgo ideológico.
Sus egresados carecen de formación
disciplinar pero tienen su nombra-
miento "legal"... entre comillas Eso es
un problema y otro problema
adicional son los salarios pírricos para
todos los problemas que debemos
enfrentar, y las condiciones precarias
en las que uno trabaja. Ese sueldito no
da para cubrir cursos de
actualización.

De esta reflexión se desprende la subcategoría desvalorización de la formación docente, en su doble aspecto tanto profesional como remunerativa. No obstante, el reconocimiento de desigualdades en cuanto a competencias docentes dependiendo de la institución formadora, asoma como una fortaleza para la Universidad Pedagógica.

Sin embargo, en la acotación acerca de docentes en servicio con o sin formación pedagógica y severas debilidades en conocimientos disciplinares, se interpreta una desvalorización de la profesión docente, pues se piensa que cualquiera puede ser profesor, lo cual refleja la baja valoración que la sociedad tiene de la docencia. La consideran una labor rutinaria, intra-áulica, cuya ejecución no requiere mayor cualificación, experticia, ni certificación de quien la ejerce. Sin embargo, eso ocurre, en muchos casos con el aval de las autoridades por el marcado sesgo de intereses ideológicos que ha conducido a la creación de instituciones educativas paralelas al servicio de intereses 
muy alejados de la pedagogía.

Esta desvalorización docente es analizada, entre otros, por Brunner (2011) y Ravelo (2018) quienes califican el salario del docente como poco competitivo lo que significa ausencia de estímulo, razón por la cual los profesores jóvenes prefieran emigrar en busca de alternativas para su desarrollo profesional.

La realidad educativa que afronta esta sociedad, requiere de cono-cimientos en liderazgo social, desarrollo humano, capital social para intervenir en las comunidades y nada de eso estaba en mi pensum de estudio- En eso la universidad debe revisarse.

A partir de esta unidad de información surge la subcategoría nuevas demandas en la formación del docente. Ante la carencia de estrategias para abordar la complejidad de las prácticas educativas cotidianas, cuando la formación inicial no considera las vías para afrontar muchos de estos graves problemas, la socialización laboral, entendida como la educación no intencional, la transmitida involuntariamente por el entorno familiar y social, se constituye en la vía mediadora. Es el conocimiento empírico, el adquirido con la experiencia, al cual se refiere el informante. La sistematización de las experiencias expresa Rodríguez (2009), permite generar conocimientos desde las prácticas [...] para tomar decisiones, lo que conviene hacer a la luz de las lecciones aprendidas. No obstante, este camino sería menos tortuoso si los docentes contaran con la preparación teóricopráctica al respecto, en su formación inicial o se tomara en consideración para la formación permanente. Es justamente a través de la reflexión del egresado que las instituciones educativas, argumenta Moncada (2011), pueden hacer seguimientos de sus políticas de gestión $\mathrm{y}$, los resultados pueden utilizarse para introducir mejoras y nuevas orientaciones en los programas educativos.

En este caso, es muy pertinente este llamado, pues el informante insta a la universidad a revisarse justamente cuando la intervención en las comunidades, está prevista en la Ley de Servicio Comunitario (2005) la cual debe cumplirse en 120 horas y es requisito de grado.

Para orientar muchos de los problemas de los estudiantes, el profesor tiene que hacer uso de lo que trae de su familia, porque uno ya es una persona con valores cuando llega a la universidad, y dependiendo de eso, puede afrontar, en parte, las situaciones para los que no nos prepara la Universidad, y no dejarse vencer por las circunstancias.

La verbalización refleja la alta valoración que el docente tiene de sí mismo, como una persona de aquilatados valores adquiridos en el núcleo familiar pues los valores generan creencias y estas a su vez, comportamientos. En ausencia de la preparación en la formación inicial para enfrentar situaciones laborales adversas e inesperadas que se plantean en las instituciones educativas, el egresado sólo cuenta con las competencias personales, vivencias propias, ingenio, creatividad, iniciativa para liderarlas, y lo hará, de acuerdo a los valores inculcados desde su entorno familiar. Se trata del desarrollo de la autonomía y de la responsabilidad adquirida en el marco de las relaciones de familia, al ser éste el primer escenario donde se socializa la persona. Posteriormente, a lo largo del desarrollo social, ésta continúa con el cultivo de los valores y con ellos llega a la universidad donde se consolidan hasta constituirse en parte de sí misma. Nótese que el informante hace énfasis en la ausencia de preparación por parte de la Universidad, para 
enfrentar ciertas situaciones. Al respecto López (2011) señala que es responsabilidad de los docentes mostrar en su vida cotidiana los valores en los cuales cree para estimular el desarrollo de actitudes correspondientes.

Nos faltan herramientas para enfrentarnos al día a día de un aula, por ejemplo cómo crear en mis estudiantes el interés en el contenido que se imparte. ;Que tarea tan difícil! Te das cuenta que prefieren pasar horas ha-blando en el Facebook que investigar o leer sobre algún tópico indicado por el profesor.

De la verbalización del egresado surge la subcategoría acompañamiento para utilizar las nuevas tecnologías. Esta implica una realidad relacionada con el reto del docente de hoy: hacer de las nuevas tecnologías unas aliadas para captar el interés del estudiante y aprovechar la penetración de estos nuevos medios tecnológicos, en los nativos digitales, para aprender a través del entretenimiento. Entre estas se pueden mencionar, las aplicaciones disponibles en el mercado para facilitar el aprendizaje de la Tabla Periódica en química, dibujo, los libros digitales. Con relación a la utilización de las TIC, Lamelli (2017) postula que es necesario implementar actividades de aprendizaje innovadoras, estrategias concretas centradas en el estudiante a fin de impulsar el desarrollo de habilidades cognitivas pues la sola presencia de las nuevas tecnologías, no es garantía de nuevas prácticas. Es necesario disponer de contenidos dirigidos a los docentes con programas y aplicaciones educativas para su propio aprendizaje y el de sus estudiantes enmarcados en una actualización permanente.
En la universidad existe la intención por desarrollar la formación docente actualizada y de calidad. Sin embargo, tal intento resulta fallido debido a la utilización de metodologías desactualizadas, o con objetivos en sus programas que no se cumplen o con planes de evaluación de los cuales no se puede extraer un provecho real.

El discurso del informante permite proponer la subcategoría la intención de los postulados curriculares se desvanecen en la ejecución. Es la viva expresión entre el ser y deber ser, entre la intención versus la realidad, que dejan al descubierto fisuras en torno a la calidad de la formación docente. De acuerdo a la UNESCO (1997) la calidad universitaria se define por la adecuación del ser y quehacer de la educación superior a su deber ser. El primero enfatiza en los mecanismos de las instituciones para asegurar la calidad, el ser se refiere a los logros actuales y el deber ser a los propósitos que la misma institución se ha trazado lograr y es justamente en esta triangulación donde las instituciones deben interrogarse para responder si la intención se hace tangible en la realidad.

Los estudiantes caen en el tedio y el marasmo académico no sólo por la desactualización de los contenidos sino también por los métodos de enseñanza de otros tiempos. Mora (2004), propone como alternativa la enseñanza por proyectos, las aplicaciones, la resolución de problemas, las estaciones de trabajo y aprendizaje, entre otros, cuyo denominador común es la cooperación y participación de los integrantes de diferentes disciplinas.

En consecuencia, la universidad formadora de formadores debe abocarse a la transformación de los programas de formación para dar respuestas favorables a los docentes que manifiestan estar 
conscientes de su insuficiente preparación profesional pues no siempre las intenciones curriculares se cristalizan en la realidad. Es así como se devela la importancia de la mediación de las experiencias, a fin de contrastar los logros, exponer a la luz pública las competencias docentes y comparar con los propósitos fundamentales a fin de hacer los correctivos necesarios.

El caso de los programas de Educación para la Paz, Empoderamiento y Desarrollo sustentable, son ejemplos de intentos por ayudar a mejorar la formación docente, más allá de lo escrito en los documentos, pues proporcionan herramientas a los docentes para constituirse en agentes de cambio social.

En mi formación docente influyó la pasión, la ética y la entrega que algunos, no todos, de mis profesores transmitieron en el aula, excelentes en su área disciplinar, porque tienes que dominar lo que vas a enseñar y que sabían transmitir los contenidos, además, se aprendía de las evaluaciones porque recibías observaciones que te ayudaban a mejorar, esa evaluación formativa tan importante.

La formación docente depende de variados factores, de acuerdo a la acción reflexiva del informante, pero la actuación de los profesores conforma una pieza fundamental. De aquí surge la subcategoría modelaje docente, la cual se puede relacionar con la Teoría Social del Aprendizaje de Bandura (1977) basada en el proceso de modelaje cuyo postulado señala que guardamos, el comportamiento del modelo en forma de imágenes mentales o descripciones verbales y una vez "archivadas", podemos hacer resurgir la imagen o reproducirla con nuestro propio comportamiento. De este modo, se explica que los profesores sirvan de modelo para la adquisición de comportamientos, pues el docente es la referencia a ser tomada en cuenta para sus acciones tal y como es verbalizado por los informantes.

La verbalización puede verse en tres planos: El egresado hace énfasis con las palabras No Todos para acentuar que no es la norma. Por otra parte, pondera de sus formadores el dominio del área disciplinar como requisito para enseñar y recordamos la conocida sentencia: No se puede enseñar lo que se desconoce. Adicionalmente, valora al docente como un recurso humano valioso y decisivo en la formación cuando además de ser virtuoso en su disciplina, también es ético, aplica variadas estrategias de aprendizaje y de evaluación para motivar al logro, centra su atención en el estudiante al señalarle sus debilidades, errores con la finalidad de superarlos. Lo expuesto encuentra su apoyo en López (2009), cuando sustenta que la fortaleza de la evaluación formativa estriba en ser orientadora, mediadora e individual.

Fue gracias a un profesor con quien descubrí el placer de indagar trabajar con instrumentos, ser ordenado y disciplinado, insistir cuando los resultados eran incorrectos, ver más allá de los datos, ser crítico ante los resultados, trabajar en equipo, respetar las opiniones de los otros, participar con ponencias en eventos de investigación, escribir artículos y a entender que todo eso forma parte de los atributos de un investigador.

La unidad de información nos revela como subcategoría modelaje para la formación del investigador entendido como la influencia docente para marcar el rumbo en sus estudiantes, acerca de las diversas vías en la generación de nuevos conocimientos Esto subraya una vez más la influencia del modelaje docente, en este caso, en el plano 
investigativo pero acompañado de valores tales como orden, disciplina, tolerancia, autocrítica.

Ante los acelerados cambios tecnológicos y humanísticos de un mundo globalizado corresponde a los docentes desarrollar en los estudiantes sus competencias interpretativas, investigativas y explicativas en un contexto donde la universidad, orientada hacia la indagación y la búsqueda del saber, produce el conocimiento, lo organiza lo difunde y lo socializa. En la actualidad, se requiere de un profesional que lleve a las aulas su producción intelectual y estimule en sus estudiantes la inquietud por la investigación a fin de evitar la caducidad y el marasmo intelectual.

No por azar, los nuevos paradigmas curriculares integran docencia, investigación y extensión. Sin duda en el campo educativo, la investigación es una vía para resolver múltiples problemas y por tanto es necesario propiciar este modelaje. En este sentido, Díaz (2006) señala que se trata de pensar y actuar en la formación de un docente investigador que desde su relación teoría-prácticareflexión, esté en capacidad de elaborar conocimientos, para ser socializados, sistematizados y ser útiles a la sociedad.

\section{Recuerdo con gratitud a una profesora de quien aprendí que no tenemos todas las respuestas y al reconocerlo ante los estudiantes no perdemos respeto sino más bien reconocimiento, pues debemos darles herramientas para que inicien la búsqueda por ellos mismos. No darles el pez, sino enseñarlos a pescar.}

Del testimonio surge la subcategoría, formar para la Autonomía, entendida como la capacidad para comprender la información, analizarla, evaluarla, relacionarla, aplicarla e integrarla a saberes nuevos con base en conocimientos previos, pues en la actualidad aprender es algo más que memorizar. De aquí que, la formación del docente en estas nuevas estrategias sea fundamental para desarrollar en el estudiante autonomía y hacerlo protagonista de su propio aprendizaje, en un mundo de incertidumbre, apelando a la motivación y a la autoestima.

En este sentido, como lo establece Pozo (2009), en concordancia con las orientaciones internacionales de los cambios en todos los niveles de la educación formal, una de las funciones del docente es promover en los estudiantes, la capacidad para gestionar sus propios aprendizajes, adoptar una autonomía creciente en su carrera académica, al disponer de herramientas intelectuales y sociales conducentes a un aprendizaje continuo a lo largo de la vida.

Esta debe ser una de las metas prioritarias, se trata entonces del docente mediador, el responsable de establecer el andamiaje de manera que el estudiante se apropie del conocimiento, tenga la capacidad de hacer la transferencia cognitiva y lo canalice hacia su entorno social en un acompañamiento pedagógico. La autonomía y la responsabilidad se entienden como valores morales y dependiendo de la calidad de su transferencia y aprendizaje, se podrá contribuir a la promoción de una ciudadanía que propicie la construcción de comunidades democráticas más justas y equitativas. Picón (2017) apunta directamente a la configuración de un sistema educativo para la formación de ciudadanos capaces de propiciar, sostener y defender un modo de vida y un sistema político basados en los valores, principios y prácticas de la democracia. 
En la universidad, encontré algunos docentes que no cumplían su horario, tenían un lenguaje peyorativo, no aceptaban críticas constructivas, las calificaciones nunca a tiempo, inexistencia de evaluación formativa, eran monótonos, los recuerdo como la antítesis de una buena imagen de la formación docente, a ellos no me quiero parecer. Emulo lo bueno, lucho contra los inconvenientes, que son muchos, me las ingenio.

Ante la reflexión del informante, pensamos en la metáfora de la resiliencia docente pues a pesar del contexto formativo desfavorable, el protagonista marca distancia de los antivalores reflejados por algunos de sus profesores y expresa con total convicción su postura. Esta actitud es denominada por Korthagen (2004) misión, lo cual se refiere a lo que está dentro de nosotros y nos mueve a hacer lo que hacemos de acuerdo al conjunto de valores que hemos asumido en la vida.

Lo anterior indica que no todo lo proporciona la Universidad, también hay una parte de la personalidad, de la individualidad para afrontar retos y superar situaciones adversas, capacidad de resiliencia, en este caso docente. El informante está convencido de la necesidad de practicar e inculcar valores a través del modelaje, aun cuando incesantemente se repite que los medios virtuales de enseñanza conducen a la desterritorialización de la misma. Sin embargo, entre los factores o variables que presentan mayor incidencia en el logro de aprendizajes de calidad indica López (2011), es el aportado por la interacción entre profesor y estudiante, el resto de los elementos del sistema educativo, sólo proporcionan el contexto dentro del cual se realiza dicha interacción.
¿Cuando ejerces la docencia te das cuenta que una cosa es enseñar y otra muy diferente el aprender! ¡Sí! Hay que saber cómo el estudiante aprende, si son visuales, auditivos. ¡Eso me quedó de mi formación, Claro, no es fácil, tenemos inconvenientes, pero no me dejo vencer!

El testimonio revela la subcategoría autoestima docente entendida como el conjunto de vivencias y sentimientos, generados a partir de las experiencias compartidas con personas significativas de su entorno, a través de las cuales elabora su seguridad, confianza, aceptación y percepción de ser competente además de exitoso ante las actividades desarrolladas. La reflexión implica una fuerte motivación que le conduce a seleccionar estrategias generadoras de un crecimiento y éxito personal.

El docente al considerar que enseñanza y aprendizaje implican realidades diferentes, se esfuerza, a pesar de los obstáculos, por implementar variadas estrategias de enseñanza, imprescindibles en el mundo cada vez más complejo del siglo XXI, a fin de potenciar el aprendizaje de los estudiantes. Las distintas estrategias tienen por finalidad llegar con mayor facilidad a través de los distintos canales cerebrales de los aprendices de acuerdo a su desarrollo cognitivo. Como lo argumenta Cossio (2017), los mismos contenidos se podrían presentar de formas diversas a fin de aprovechar las fortalezas del estudiante, permitiendo asimilarla según sus capacidades y habilidades cognoscitivas. Las alianzas con sus pares le permiten incursionar en la integración de los contenidos, ampliar la red del conocimiento, aprovechar las oportunidades, ser creativo, emprendedor y trabajar en equipo. 
Hay que, estar al día, porque la nueva información nos arropa y si nos descuidamos nos quedamos como analfabetos funcionales. Entonces, ¿Cómo quedamos ante nuestros estudiantes? Fíjese, empecé en un liceo medianamente dotado, pero nadie utilizaba esos materiales por no saber $o$ no querer hacerlo. Como en el pregrado había cursado materias donde manejé todo eso, lo organicé y ahora, todo se utiliza.

La interrogante desplegada por el docente pone de relieve el autoconcepto, como subcategoría, entendida como el punto de vista que nos atribuimos lo cual nos conduce al compromiso con la profesión escogida, dando como resultado la identidad profesional, definida por Korthagen (2004), como las creencias sobre uno mismo, más los significados que otros nos atribuyen. En este caso, el docente se asume como un profesional cuya actualización tiene prioridad por respeto a sí mismo y para no defraudar la imagen docente ante sus estudiantes. El informante ante la consciencia de la caducidad del conocimiento, declara la voluntad de querer estar actualizado en su especialidad. En este sentido, los estudios de postgrado se constituyen en una posibilidad para influir de manera positiva en la ejecución del trabajo pedagógico y mejorar el rendimiento académico de los estudiantes. El autoconcepto, el compromiso y la identidad profesional, se constituyen en apalancamientos que le permiten resolver, de manera satisfactoria, la paradoja de laborar en un centro educativo dotado de recursos pero sin considerar a los docentes, con lo cual los objetivos de la dotación se desvanecen.

\section{Síntesis conceptual de los hallazgos}

Los egresados se expresan libremente de manera diáfana y espontánea, esto les permite profundizar en la reflexión y señalar desde su perspectiva, lo que consideran los aciertos y desaciertos en la formación recibida. A lo largo de las verbalizaciones de los entrevistados, se va configurando todo un mundo de sentido subjetivo donde se develan las necesidades académicas, personales, laborales, los sentimientos, los valores, los anhelos, entre otros, que además de dar cuenta de las creencias implícitas de los docentes, también sugieren la acción transformadora de sus prácticas y sirven para orientar al investigador en la interpretación.

Así, de la codificación abierta aplicada al ejercicio reflexivo de los egresados, emergen diez y seis subcategorías cuyos contenidos reflejan que su formación docente está marcada por la multiplicidad de funciones y alejada de la cruda realidad social del país, caracterizada por estar inmersa en la violencia, en las desigualdades lo cual hace muy complejo el acto educativo. Además, el egresado vive la desvalorización de la formación docente al protagonizar la incongruencia del discurso entre la importancia de la función docente y el efectivo reconocimiento profesional y social en términos de remuneración para promover un educador competente, motivado y bien valorado socialmente. De este modo, la intención de los postulados curriculares universitarios se desvanece en la ejecución. Por esto, en muchos casos, cuando la formación de la Universidad es insuficiente, el egresado se apoya en la fuerza de los valores familiares para orientarse en la resolución de los problemas del contexto laboral.

Todo lo cual evidencia la necesidad de actualización docente en un país caracterizado por políticas educativas erráticas, mal implementadas, apoyo muy débil para actualización que conduce a una escasa formación permanente por una parte, y por la otra una infraestructura deficiente, 
equipamiento inconcluso de las instituciones educativas, carencia de recursos didácticos, o como también ocurre, de manera paradójica, centros educativos dotados sin el respectivo acompañamiento a los docentes. También reclaman otros aspectos, uno de ellos es el acompañamiento necesario para utilizar las herramientas digitales a fin de enfrentar la obsolescencia del conocimiento además de mejorar y facilitar los procesos de enseñanza $\mathrm{y}$ de aprendizaje en un mundo digitalizado y sin fronteras. Destacan como fortalezas de su formación el dominio disciplinar, pero sólo cuando ejercen en las áreas en las que han sido formados, y la influencia de sus profesores cuyo modelaje les ha incentivado a seguir la ruta del docente investigador. Igualmente admiten los beneficios cognitivos de orden valorativo adquiridos por estrategias extra áulicas como el trabajo de campo Ante la avalancha, cuyas experiencias constituyen vivencias de vocabulario técnico. Reconocen que se debe formar para la autonomía con estrategias de aprendizaje para aprender a gestionar la incertidumbre del siglo XXI. Se percibe de los relatos, que los entrevistados actúan de acuerdo a sus valores los cuales les imponen un comportamiento ético, por ello utilizamos la alegoría resiliencia docente pues a pesar de las limitaciones del contexto, de los escollos encontrados en la ruta elegida, al docente lo mueve: la vocación, la motivación, el autoconcepto, la identidad pedagógica; al estar convencido de hacer un trabajo digno, valioso de servicio social en el cual se ayuda a los hijos de otros a crecer, superar sus dificultades, emprender sus proyectos de vida. En este sentido, el docente pone en escena las dimensiones fundamentales personales: el ser, el saber, el saber hacer y el emprender.

Al aplicar la codificación axial, a las diez y seis subcategorías, estas quedan reducidas a tres categorías emergentes que constituyen las referencias a partir de las cuales los egresados se han reconocido a partir de sus testimonios a) referencias ontológicas, b) referencias epistemológicas, d) referencias axiológicas. Entre las primeras incluimos: Multiplicidad de funciones, formación alejada de la cruda realidad y la intención curricular desdibujada en el camino. Entre las epistemológicas ubicamos: El dominio disciplinar, acompañamiento para utilizar las herramientas digitales, docente investigador, vivencias de vocabulario técnico, Formar para la autonomía. Finalmente, entre las axiológicas agrupamos: La vocación, la motivación, el autoconcepto, la identidad pedagógica, la fuerza de los valores familiares, la resiliencia docente, el modelaje en la formación del docente investigador, desvalorización de la formación docente.

La interpretación del contexto de éstas, nos confirma la necesidad de establecer modificaciones, correctivos resignificaciones $\mathrm{y}$ en un ejercicio de mayor abstracción de codificación selectiva arribamos a la categoría central: Nuevas coordenadas para un nuevo mapa pedagógico.

Del discurso del egresado se desprende que el nuevo mapa pedagógico de los programas de formación inicial docente debe incluir el manejo de las herramientas necesarias para enfrentar la variada gama de los severos problemas del mundo laboral docente actual: drogas, alcohol, violencia, discriminación, entre otros.

El convulsionado contexto social en el que se encuentra inmerso el mundo educativo actual requiere de un profesional de la docencia preparado, más y mejor, en todos los terrenos de la vida, pues, además de propiciar el desarrollo del talento de sus estudiantes también debe contribuir a forjar en estos, la construcción de un conocimiento científico basado en valores necesarios para vivir, convivir y ser emprendedores ante escenarios 
cambiantes a corto plazo. Es necesario, además de asimilar los conocimientos, utilizarlos efectivamente dentro y fuera de la institución educativa, de acuerdo con las exigencias de los distintos contextos como expresión de ciudadanos en la búsqueda de una calidad de vida mejor a la existente.

Es imperativo en el nuevo mapa, actuar de manera responsable y honesta con el medio ambiente, inserto en un planeta cuyos graves problemas ponen en peligro la supervivencia de la especie humana. Todo lo cual requiere de ejercitación en el diálogo y la negociación para mantener un desarrollo sustentable. Esto impone una transformación en nuestras rutinas docentes a fin de replantearnos continuamente la vigencia, la importancia y la responsabilidad de nuestra función en la sociedad de hoy. Adicionalmente, se requiere de un docente creativo para propiciar entornos interactivos de enseñanza-aprendizaje, donde el estudiante sea capaz tanto de transmitir como de recibir información, transitando una vía didáctica, de transferencia de conocimiento centrado en el estudiante. En este sentido, las TIC deben utilizarse como un recurso para ayudar a diseñar espacios diferentes y atractivos para el aprendizaje, reforzar el emprendimiento y creatividad. Por tanto es necesario conocer las necesidades de los egresados para acompañarlos en la formación permanente

\section{CONCLUSIONES DESDE LA PROVISIO- NALIDAD}

El estudio de las reflexiones de los egresados en torno a su formación docente, se constituye en fuente de información válida que puede servir en insumo para las instituciones formadoras de formadores a fin de mejorar la formación impartida. El discurso reflexivo del egresado en un nuevo mapa pedagógico lo podemos resumir en cuatro instancias, la formación docente requerida para: a) Orientar satisfactoriamente los problemas del contexto educativo en la cruda realidad social donde le corresponde laborar b) Utilizar adecuadamente las herramientas tecnológicas para facilitar los procesos de enseñanza y de aprendizaje c) Enfrentar la obsolescencia del conocimiento desde una referencia axiológica, y d) Formar desde la civilidad apegados a los valores de democracia y libertad en la búsqueda de la dignificación de la profesión docente.

\section{REFERENCIAS}

Azpúrua, F. (2004). El interaccionismo simbólico y la fenomenología social: Sus principales contribuciones a la etnosociología. Arbitraje (2) 3, 63

Bozu, Z e Imbernón, F. (2016). El presente y el futuro de la formación permanente docente del profesorado universitario. Educación y Ciencia, Vol $5 \mathrm{~N}^{\circ} 45$

Brunner, J. J. (2011). Informe Educación Superior en Iberoamérica José Joaquín | editor coordinador. Informe 2011 Primera edición: Centro Interuniversitario de Desarrollo (CINDA) - Universidad. Santiago de Chile

Cossio, C. (2017).Inteligencias múltiples en estudiantes de Educación Superior. Universidad Mayor de San Marcos. Lima Perú. Tesis Doctoral

Dewey J. (1989).Cómo pensamos. Paidós: Barcelona

Díaz, V. (2006). Formación Docente, Práctica Pedagógica y Saber Pedagógico. Laurus. Revista de Educación. Año 12. Número Extraordinario. pp.88-103. Universidad Experimental Pedagógica Libertador. Caracas

Gadamer, H. (2000). Sobre los que enseñan y los que aprenden. En La herencia europea. Paidós: Barcelona

Jiménez Puello, J. (2014). Estudio sobre los estándares TIC en educación en los futuros docentes de la Facultad de Educación de la Universidad Complutense de Madrid Memoria para optar al Grado 
de Doctor

Korthagen, E. (2004). In search of the essence of a good teacher: Towards a more holistic approach in teacher education. En Teaching and Teacher Education, 20, pp. 77

Lamelli, Z. (2017).Estrategias didácticas innovadoras y motivación para el aprendizaje según estudios de psicología En: Estrategias didácticas en el contexto de la complejidad Ediciones XIV Jornadas de Investigación Educativa y $\mathrm{V}$ Congreso Internacional de Educación UCV

Ley de Servicio Comunitario del estudiante de Educación Superior. (2005). República Bolivariana de Venezuela. Gaceta Oficial $\mathrm{N}^{\circ} 38272$

López P, V. (2009). Evaluación formativa y comparada en Educación Superior. Narcea: Madrid

López Benedí, J. (2011).La educación en valores como recurso para la formación del profesorado. Tesis doctoral. Universidad Autónoma de Madrid.

Mora, D. (2004). Aspectos pedagógicos y didácticos sobre el método de proyectos. Tópicos en Educación Matemática. Grupo de Investigación y Difusión sobre Educación Matemática GIDEM. Imprenta Universitaria. Universidad Central de Venezuela

Moncada, J. (2011). Modelo Educativo basado en competencias. Trillas: México.

Rodríguez del Rey, M. y Rodríguez Morales, A. (2015). La formación del docente universitario. Concepciones teóricas y metodológicas. Revista Universidad y Sociedad Vol.7 $\mathrm{N}^{\circ} .1$

Picón, G. (2018). Ciencia, Democracia y Educación. Revista Educación Superior y Sociedad Colección 25. Aniversario Vol. $24 \mathrm{~N}^{\circ} 24$.Instituto Internacional de
Unesco para la Educación Superior en América (IESALC)

Pozo, J I. (2009). Psicología del aprendizaje. La formación en competencias. Barcelona: Libros Aula Magna: Madrid

Rodríguez, N. (2009). Asesoramiento en aula: Hacia un modelo de mejora profesional de docentes. Revista de Pedagogía. Vol. 30. $\mathrm{N}^{\circ}$ 86. Escuela de Educación. U.C.V. $135-160$

Ruiz R, Sequeira V. (2010) Estudio de seguimiento de egresados y empleadores de formación inicial docente. Patria Grande Revista Centroamericana de Educación Vol 1, No.1

Strauss, A y Corbin, J. (2002). Bases de la investigación cualitativa. Técnicas y procedimientos para desarrollar la Teoría Fundamentada. Universidad de Antioquía. Colombia

Taylor, S y Bogdan, R. (1987). Introducción a los métodos cualitativos de investigación. Paidós: Buenos Aires

UNESCO. (1997). La Educación Superior en el Siglo XXI. Visión de América Latina y el Caribe. CRESALC/UNESCO. Caracas. Autor

UNESCO. (2009). Conferencia Mundial sobre la Educación Superior La nueva dinámica de la educación superior y la investigación para el cambio social y el desarrollo. Paris. Autor

Van Manen, J. (1997). Linking ways of knowing with ways of being practical. Curriculum Inquiry, Vol 6, $\mathrm{N}^{\circ} 3$ pp. 205228

Yegres, A. (2018). Educación Democrática y de calidad: un mundo que ganar en el ámbito de las universidades venezolanas Educación Superior y Sociedad Colección 25을 Aniversario Vol. $24 \mathrm{~N}^{\circ} 24$. Unesco (IESALC) 